\title{
HMGB1 levels are increased in lesional skin and sera in patients with erythrodermic cutaneous T-cell lymphoma
}

Naoyuki Senda ${ }^{1}$, Tomomitsu Miyagaki ${ }^{1}$, Hiroaki Kamijo ${ }^{1}$, Tomonori Oka ${ }^{1}$, Naomi Takahashi ${ }^{1}$, Hiraku Suga ${ }^{1}$ Makoto Sugaya ${ }^{1,2}$ and Shinichi Sato ${ }^{1}$

${ }^{1}$ Department of Dermatology, the University of Tokyo Graduate School of Medicine, Tokyo, Japan

2 Department of Dermatology, International University of Health and Welfare, Chiba, Japan

\section{INTRODUCTION}

High mobility group box-1 (HMGB1) is a nonhistone nuclear protein to stabilize nucleosome and to modulate transcriptional activity. HMGB1 is released to extracellular fluid actively by inflammatory stimulation and passively by cell death. Extracellular HMGB1 acts as a danger signal, that induces migration, proliferation, and activation of various cells. There are emerging reports describing that HMGB1-mediated tissue damage and inflammation are associated with development of various inflammatory and autoimmune diseases and malignancies. The clinical significance of HMGB1 has also been shown in various lymphomas.

\section{OBJECTIVE}

To investigate the roles of HMGB1 in CTCL.

\section{MATERIALS AND METHODS}

Serum HMGB1 levels were measured in 22 patients with CTCL and 7 healthy controls by ELISA. HMGB1 mRNA expression in CTCL skin $(n=21)$ and in normal skin $(n=$ 11) was examined by quantitative RT-PCR. HMGB1 expression in lesional skin was also examined by immunohistochemistry.

\section{RESULTS}

(a)

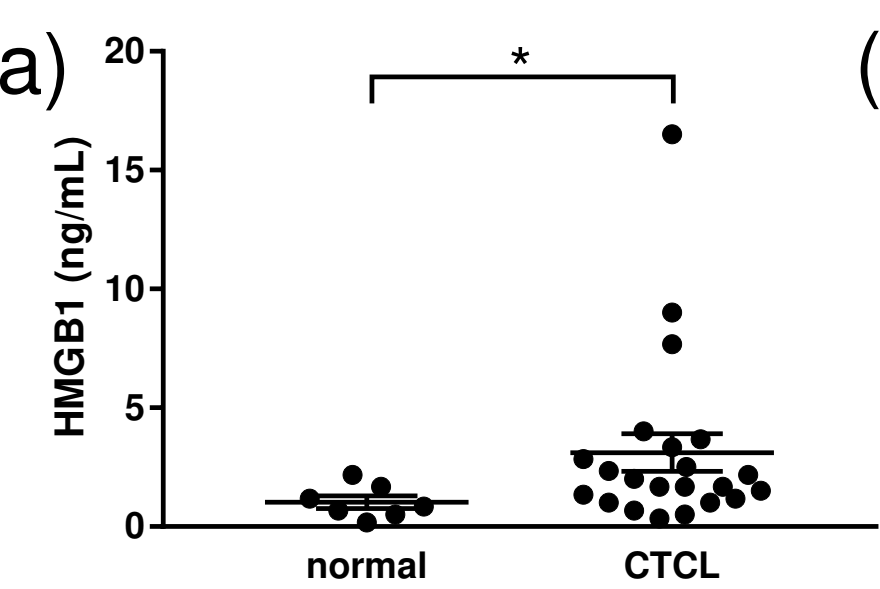

(b) ${ }^{2}$

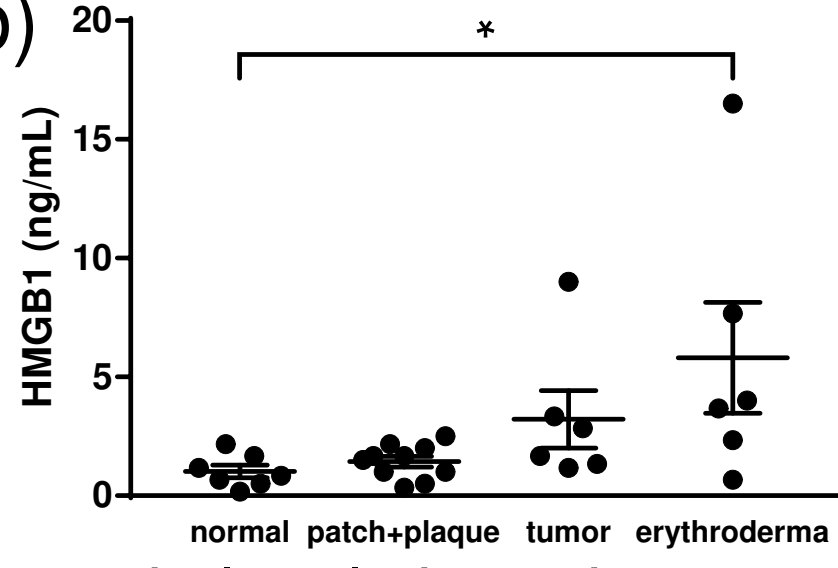

Figure 1. (a) Serum HMGB1 protein levels in patients with cutaneous $T$ cell lymphoma (CTCL) and normal controls. (b) Serum HMGB1 protein levels in patients with CTCL classified with types of skin and normal controls.
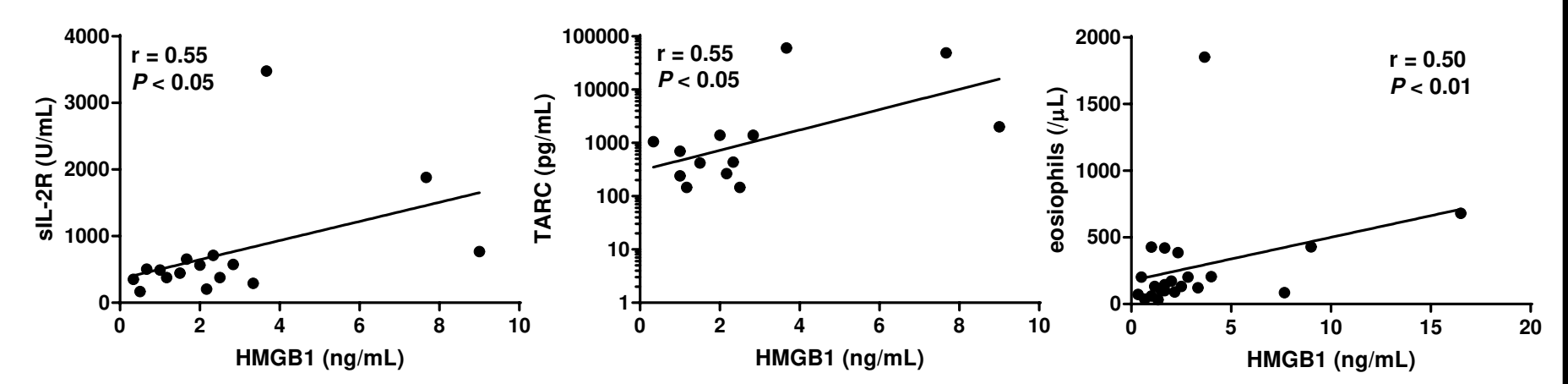

Figure 2. Correlations between serum HMGB1 levels and serum sIL-2R levels, serum TARC levels, and the number of eosinophils in peripheral blood in patients with CTCL.
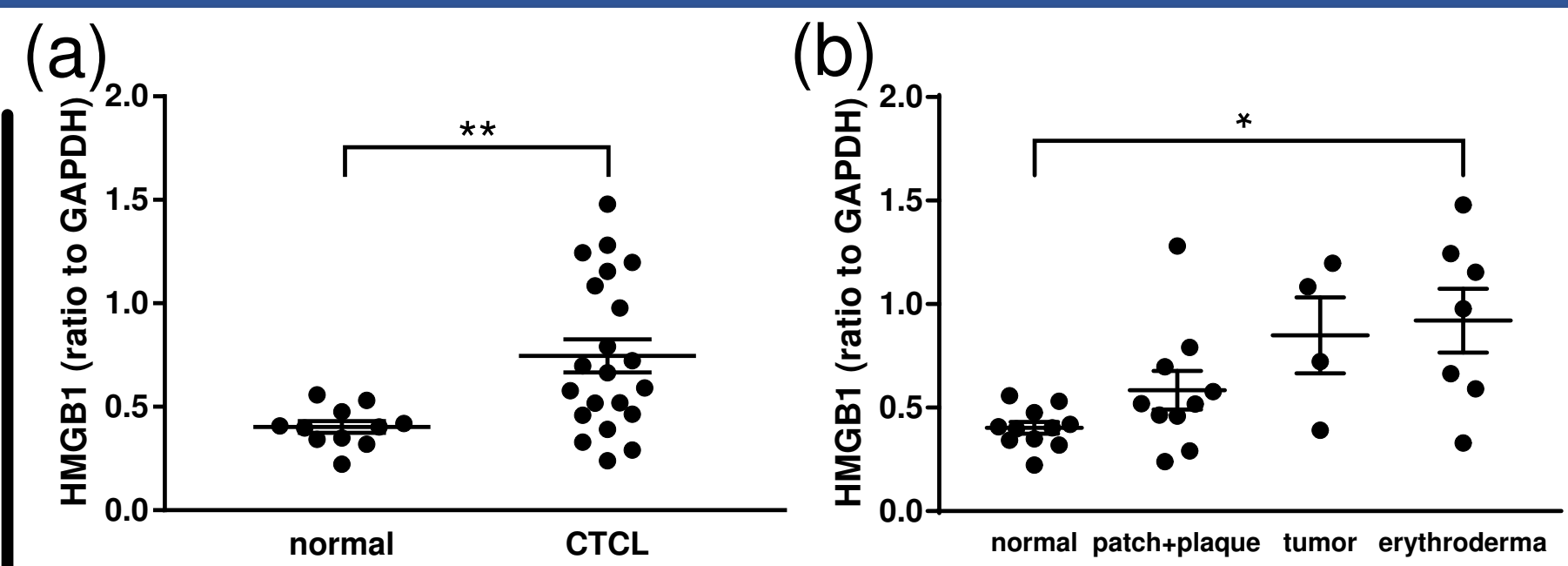

Figure 3. (a) HMGB1 mRNA expression levels in lesional skin of CTCL and normal control skin. (b) HMGB1 mRNA expression levels in lesional skin of CTCL classified with types of skin and normal control skin.
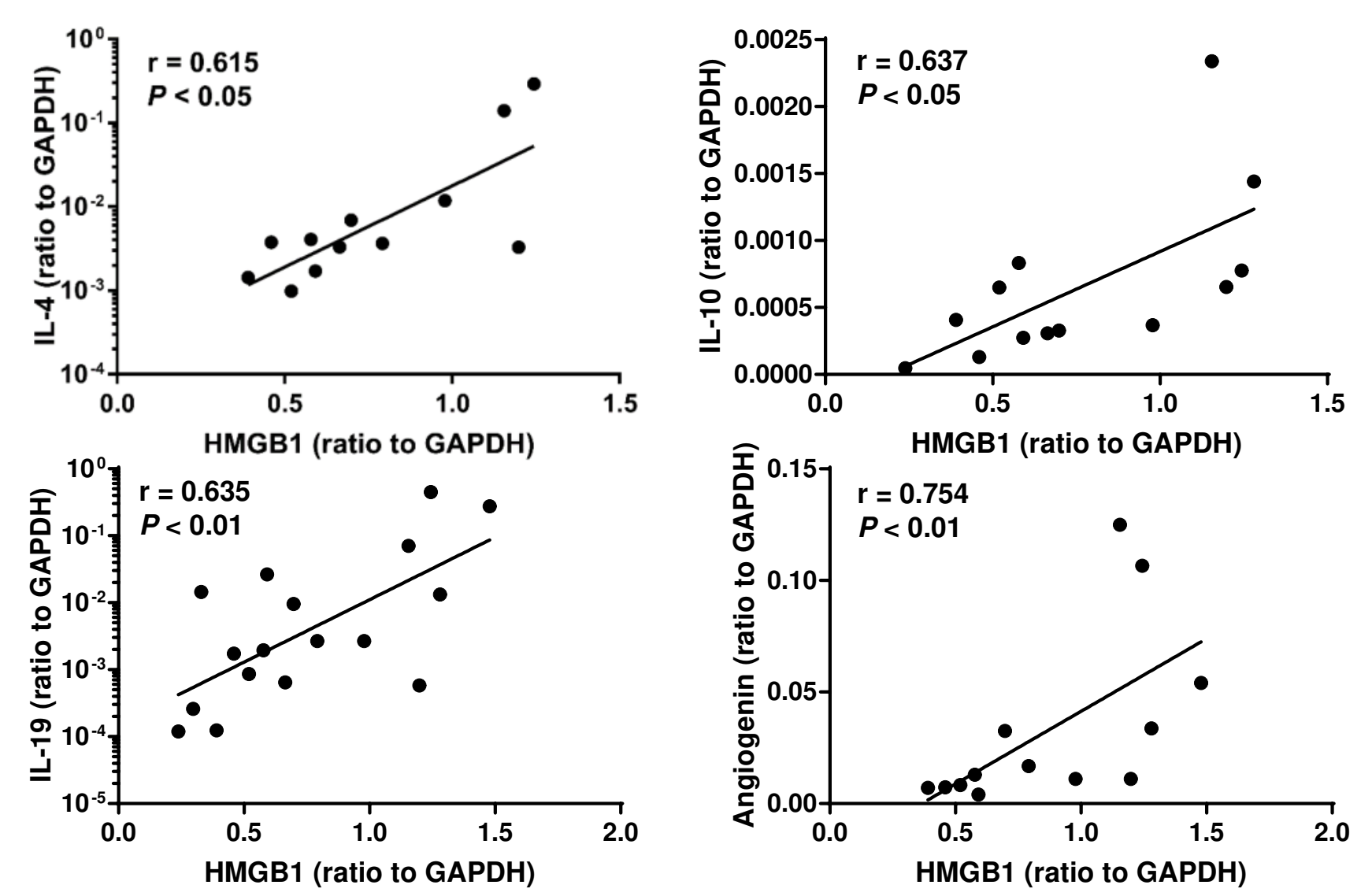

Figure 4. Correlations of mRNA expression levels between HMGB1 and IL-4, IL-10, IL-19, and angiogenin in lesional skin of CTCL.

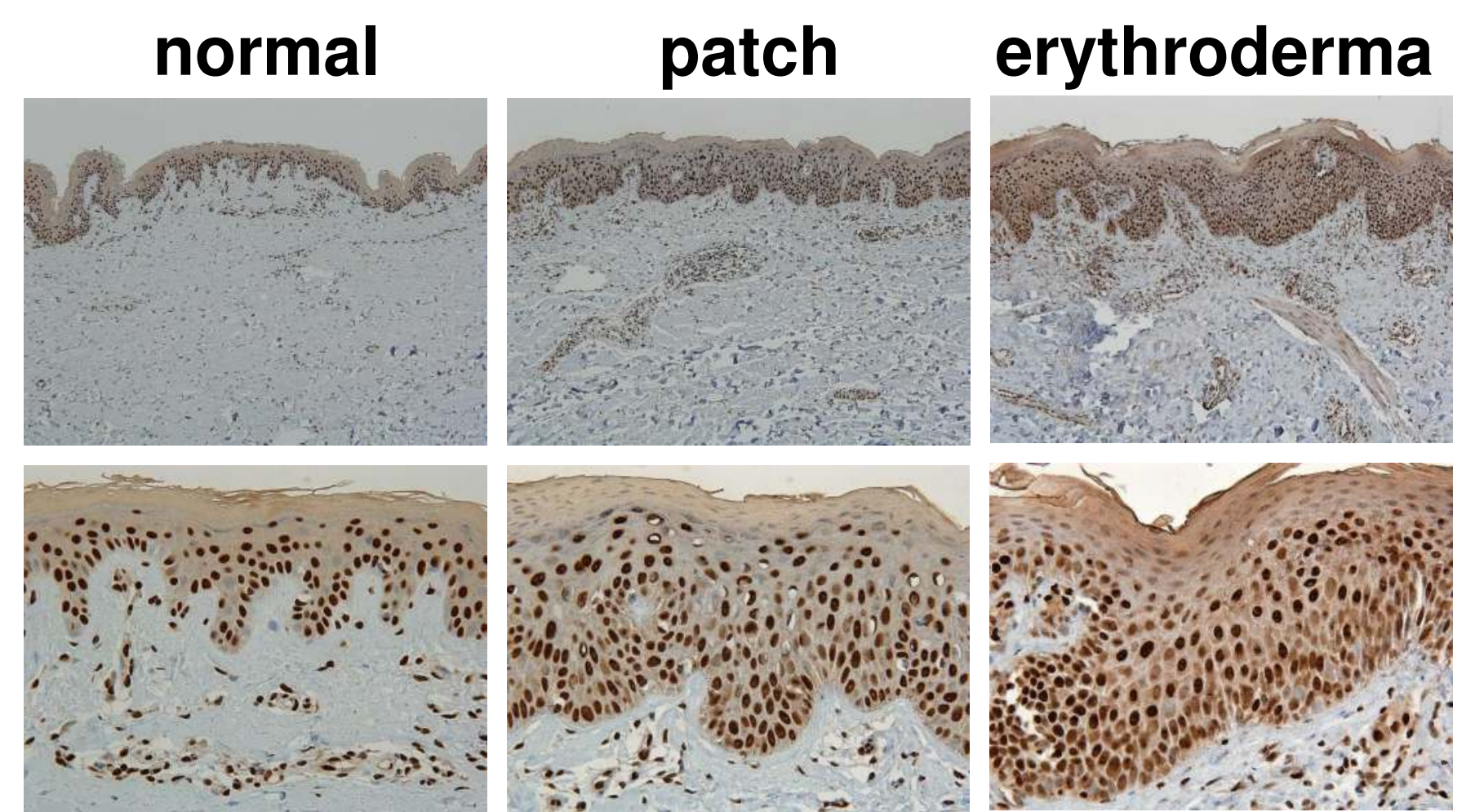

Figure 5. Immunohistochemistry for HMGB1 in normal skin, lesional skin of patch MF and lesional skin of erythrodermic MF.

\section{CONCLUSION}

HMGB1 was highly expressed in both serum and lesional skin of CTCL, especially of erythrodermic CTCL. HMGB1- mediated signaling can contribute to the development of CTCL through Th2 polarization, promoting angiogenesis, and chemotaxis of tumor cells. 